\title{
A new original nutraceutical formulation ameliorates the effect of Tadalafil on clinical score and cGMP accumulation
}

\author{
Vincenzo Mirone ${ }^{1}$, Luigi Napolitano ${ }^{1}$, Roberta D'Emmanuele di Villa Bianca ${ }^{2}$, Emma Mitidieri ${ }^{2}$, \\ Raffaella Sorrentino ${ }^{2}$, Arianna Vanelli ${ }^{3}$, Domenico Vanacore ${ }^{2}$, Carlotta Turnaturi ${ }^{2}$, \\ Roberto La Rocca ${ }^{1}$, Giuseppe Celentano ${ }^{1}$, Davide Arcaniolo ${ }^{4}$, Giuseppe Cirino ${ }^{2}$ \\ ${ }^{1}$ Department of Neurosciences, Sciences of Reproduction, and Odontostomatology, University of Naples Federico II, Naples, Italy; \\ ${ }^{2}$ Department of Pharmacy, School of Medicine and Surgery, University of Naples, Federico II, Naples, Italy; \\ ${ }^{3}$ Responsabile Ricerca e Sviluppo Nutrilinea SRL, Varese, Italy; \\ ${ }^{4}$ Urology Unit, Department of Woman Child and of General and Specialist Surgery, University of Campania "Luigi Vanvitelli", \\ Naples, Italy.
}

\begin{abstract}
Summary Objective: To assess the efficacy of the combination of Tadalafil $5 \mathrm{mg}$ and nutritional supplements composed by Panax ginseng, Moringa Oleifera and Rutin on erectile function in men with mild and moderate vasculogenic ED.

Methods: we prospectively enrolled 86 patients divided into two groups A (45), B (33) in this multicenter randomized, doubleblind, placebo-controlled trial. Drop out was 8 patients (3 patients in group A and 5 in Group B). At screening visit patients underwent clinical examination, blood test (hormonal and metabolic profile) and filled out the IIEF-5 questionnaire and the SEP-2, SEP-3. Patients were randomized by a computergenerated list to receive either Tadalafil $5 \mathrm{mg}$ once daily plus nutritional supplement once daily (group A) or Tadalafil $5 \mathrm{mg}$ plus placebo with the same administration schedule (group B) for 3 months. Blood samples, IIEF-5, SEP-2 and SEP-3 have been collected again after 3 months. cGMP was measured in platelets of 38 patients at baseline and after one months. Results: Mean age was $59.98 \pm 6.90$ (range 38-69), mean IIEF-5 score at baseline was $13.59 \pm 3.90$. After three months of treatment, IIEF-5 score significantly improved in both groups compared to baseline (13.18 \pm 3.75 vs $20.48 \pm 2.24, p<0.0001$; $14.15 \pm 4.09$ vs $19.06 \pm 4.36, p<0.0001$, in group $A$ and group $B$ respectively). Patients treated with Tadalafil plus nutritional supplement showed a significantly higher increase in IIEF-5 score compared to those who received placebo $(7.27 \pm 2.20$ and $4.9 \pm 2.79$, respectively; $p<0.0001$;). No hormonal differences and metabolic effects were found. According cGMP result, nutritional supplements ameliorates and extends the activity of the chronic treatment.

Conclusions: IIEF-5 significant increase in group B, can be ascribed to the nutritional supplement properties and antioxidant effects of moringa oleifera, ginseng and rutin and this can enhance the endothelial NO and cGMP production.
\end{abstract}

KEY WORDS: Erectile dysfunction; PDE5; Dietary supplement; Phosphodiesterase: Natural health product.

Submitted 25 January 2021; Accepted 5 March 2021

\section{INTRODUCTION}

Erectile dysfunction (ED) has been identified as the most common sexual problem, with high prevalence and inci- dence worldwide, that affects the quality of life (QoL) of patients and their partner's. It is estimated that about 322 million men would suffer from ED global by the year 2025 (1). ED primarily affects men older than 40 years of age. ED prevalence ranges between 1-10\% in men younger than 40 years (International Consultation Committee for Sexual Medicine). The prevalence increases with age in a range from $2 \%$ to $9 \%$ in men between the ages of 40 and 49 years, $20-40 \%$ in men aged 60-69 years and $>50 \%$ in men older than 70 years (2). To the ED onset contributes several environmental and lifestyle risk factors such as diabetes mellitus, hypertension, hyperlipidaemia, obesity, metabolic syndrome, depression, smoking and limited or absence of physical exercise (3-7).

Penile erection is a complex of events controlled by vascular, hormonal and neuronal systems (8). For what concerns the vascular component the endothelium plays a major role through the nitric oxide (NO) pathway. Indeed, the activation of the NO pathway causes relaxation of smooth muscle in the penile corpus cavernous, leading to increased inflow of blood (9). NO is synthesized within the endothelium starting by endothelial nitric oxide synthase (eNOS) and activates the soluble guanylyl cyclase (sGC) that leads to the formation of cyclic guanosine monophosphate (cGMP). The cGMP levels are tightly controlled by phosphodiesterases (PDEs) (10). Nowadays, medical interventions for ED management include oral drugs, intrapenile therapies (intra-urethral suppositories and intracavernous injections) and penile prosthesis implantation (11). The most widely used therapeutic approach relies on the use of phosphodiesterase type 5 (PDE5) inhibitors (12-13). The PDE5 response rate is about $70 \%$.and it is significantly lower in difficult-to-treat subpopulations (14). Many studies have also shown that the dropout rate with PDE5 inhibitors therapy is still more than 50\% after one year (15). Emerging shreds of evidence propose an increasing role of herbal-based dietary supplements and nutraceuticals in the management of ED, for their anti-oxidant, anti-inflammatory and anti-proliferative properties.(16).

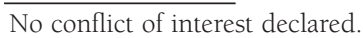


The study aims to evaluate the efficacy of the combination of Tadalafil $5 \mathrm{mg}$ and nutritional supplements composed by Panax ginseng, Moringa oleifera and rutin on erectile function in men with mild and moderate vasculogenic ED. In order to address this issue, we applied two different approaches: i) the assessment of Index of Erectile Function (IIEF) and Sexual Encounter Profile (SEP) that represent primary endpoints in clinical studies on ED (17); ii) the measurement of platelet cGMP content that represents biomarker of PDE5 activity (18-19).

\section{Methods}

The study consisted of two different phases: a clinical one and an experimental ex vivo one.

In a multicenter randomized, double-blind, placebocontrolled trial we enrolled consecutive patients with vasculogenic ED attending Urology Clinic of University of Naples "Federico II", University of Campania "Luigi Vanvitelli" and Interdepartmental Research Center for Sexual Medicine (CIRMS), University of Naples Federico II. Inclusion criteria were: patient age between 18 and 69 years; mild to moderate ED for at least 6 months with a short form of International Index of Erectile Function score (IIEF-5) $>7$ and $<22$; hypertension treated with ACE inhibitors, beta-blockers or calcium antagonists and/or type 2 diabetes treated with oral hypoglycemic agents; the patient has been in a stable sexual relationship for > 3 months. Patients have had to be naive for PDE5inhibitors treatment. Exclusion criteria were: patients who had severe ED (IIEF-5 score < 8), ED due to endocrine disorders, premature ejaculation, previous pelvic surgery, Peyronie's disease, liver or renal failure, history of myocardial infarction, cardiovascular disease, stroke, unstable angina and heart failure within the previous 6 months, intake of nitrates, diabetes mellitus on insulin therapy, dyslipidemia under drug treatment, spinal cord injuries.

At screening visit patients underwent clinical examination, blood test (hormonal and metabolic profile) and filled out the IIEF-5 questionnaire and the SEP (SEP-2: «Were you able to insert your penis into your partner's vagina?» and SEP3 «Did your erection last long enough for you to have sexual intercourse?»). Patients who met inclusion criteria were randomized by a computer-generated list to receive either Tadalafil $5 \mathrm{mg}$ once daily plus nutritional supplement once daily (group A) or Tadalafil $5 \mathrm{mg}$ plus placebo with the same administration schedule (group B) for 3 months. Blood samples, IIEF-5, SEP-2 and SEP-3 have been collected again after 3 months. All adverse events (AEs) occurred during the study period were recorded.

The study was carried out in accordance with the Declaration of Helsinki and GCP. All patients provided written informed consent. The protocol was approved by the ethical committee of Federico II University of Napoli.

\section{Nutritional supplement composition}

The nutritional supplement used for the study resulted from a combination of Panax ginseng $(500 \mathrm{mg}$ ), Moringa oleifera $(200 \mathrm{mg})$ and rutin $(50 \mathrm{mg})$. The three components were assembled in a three-layer tablet that allows different timing for the release of active ingredients.

\section{Human washed platelets}

Blood samples were collected from additional patients who met inclusion and exclusion criteria described above. These subjects received Tadalafil $5 \mathrm{mg}$ /daily plus nutritional supplement $1 \mathrm{cpr} /$ daily for one month.

The blood samples were collected before (baseline) and after treatment.

Human washed platelets were obtained by blood $(20 \mathrm{ml})$ samples collected by venipuncture. Each sample was mixed with trisodium citrate (3.8\% w/v 1:10 ratio) and then centrifuged at $150 \mathrm{xg}$ for $10 \mathrm{~min}$ to obtain plateletrich plasma (PRP) as a supernatant. Washed platelets were prepared as previously described $(18,19)$. PRP was centrifuged at $800 \times \mathrm{g}$ for $12 \mathrm{~min}$ after the addition of $1 / 10$ volume ACD solution ( $85 \mathrm{mM} \mathrm{Na3}$-citrate, $11 \mathrm{mM}$ d-glucose, $71 \mathrm{mM}$ citric acid, pH 4.4). The pellet was resuspended in $\mathrm{Ca}+2 / \mathrm{Mg}+2$-free HEPES-Tyrode buffer (134 mM NaCl, $12 \mathrm{mM} \mathrm{NaHCO}, 2.9 \mathrm{mM} \mathrm{KCl}, 0.36$ $\mathrm{mM} \mathrm{Na}_{2} \mathrm{HPO}_{4}, 5 \mathrm{mM}$ HEPES, $5 \mathrm{mM}$ glucose, $0.5 \%(\mathrm{w} / \mathrm{v})$ bovine serum albumin, pH 7.4) and adjusted to $5 \times 105$ platelets/ $\mu$ l. The platelet number was determined by using a cell counter (AcT diff 2, Instrument Laboratory, Milan, Italy).

\section{cGMP measurement}

Human washed platelets $(5 \times 105$ platelets/ $/)$ were incubated at $37^{\circ} \mathrm{C}$ with vehicle or diethylamine NONOate (DEA-NONOate, Alexis; Vinci Biochem, Vinci, Italy), a stable donor of $\mathrm{NO}$ at the concentration of 10 $\mu \mathrm{M}$ or $100 \mu \mathrm{M}$. The reaction was stopped after 30 minutes in liquid nitrogen. DEA-NONOate spontaneously dissociates in a pH-dependent, first-order process with a half-life of $2 \mathrm{~min}$ at $37^{\circ} \mathrm{C}, \mathrm{pH} 7.4$, to liberate $1.5 \mathrm{~mol}$ of NO per mole of parent compound (20).

Platelet suspensions were hydrolyzed with $\mathrm{HCl} 3.3 \mathrm{M}$. The lysates were centrifuged $(600 \times \mathrm{g}$ for $10 \mathrm{~min})$ and cGMP measured in supernatants as described in the manufacture's protocol of cGMP EIA Kit (Cayman, Vinci Biochem, Vinci, Italy) (18).

\section{RESULTS}

A total of 86 patients were enrolled in the trial. 45 patients in group A and 33 patients in group B completed the study. Mean age was $59.98 \pm 6.90$ (range 38-69), mean IIEF-5 score was $13.59 \pm 3.90$. Table 1 showed the baseline characteristics of the two groups. No differences were noticed between groups in terms of age, baseline erectile function, comorbidities, blood tests except for total cholesterol, significantly lower in group A, and liver function tests, significantly higher in group A. After three months of treatment, IIEF-5 score significantly improved in both groups compared to baseline $(13.18 \pm 3.75$ vs $20.48 \pm$ $2.24, \mathrm{p}<0,0001 ; 14.15 \pm 4.09$ vs $19.06 \pm 4.36, \mathrm{p}<$ 0.0001 , in group A and group B respectively, Figure 1). Patients treated with Tadalafil plus nutritional supplement showed a significantly higher increase in IIEF-5 score compared to those who received placebo $(7.27 \pm 2.20$ and 4.9 \pm 2.79 , respectively; $\mathrm{p}<0.0001$; Figure 1).

A total of 28 patients (36\%) completely restored their erectile function (IIEF-5 $\geq 22$ ) and no differences were noticed between the two groups (15/45 in group A vs 
Table 1.

Baseline characteristics of patients.

\begin{tabular}{|c|c|c|c|}
\hline & Group A & Group B & $P$ value \\
\hline Patients. $n$ & 45 & 33 & \\
\hline MEAN AGE (range) & $59.93 \pm 6.75(38-69)$ & $60.06 \pm 7.31(43-69)$ & 0.93 \\
\hline $\begin{array}{l}\text { IIEF-5 MEAN (range) } \\
\text { SEP2 } \\
\text { Yes } \\
\text { No }\end{array}$ & $\begin{array}{c}13.18 \pm 3.75(8-20) \\
\%(\mathrm{~N}) \\
80 \%(36) \\
20 \%(9)\end{array}$ & $\begin{array}{c}14.15 \pm 4.09(8-21) \\
\%(\mathrm{~N}) \\
66.7 \%(22) \\
33.3 \%(11)\end{array}$ & $\begin{array}{l}0.28 \\
0.18\end{array}$ \\
\hline $\begin{array}{c}\text { SEP3 } \\
\text { Yes } \\
\text { No }\end{array}$ & $\begin{array}{l}22.2 \%(10) \\
77.8 \%(35)\end{array}$ & $\begin{array}{c}27.3 \%(9) \\
72.7 \%(24)\end{array}$ & 0.60 \\
\hline $\begin{array}{l}\text { Comorbidities } \\
\text { Hypertension } \\
\text { Yes } \\
\text { No }\end{array}$ & $\begin{array}{c}\%(\mathrm{~N}) \\
97.8 \%(44) \\
2.2 \%(1)\end{array}$ & $\begin{array}{c}\%(\mathrm{~N}) \\
90.9 \%(30) \\
9.1 \%(3)\end{array}$ & 0.17 \\
\hline $\begin{array}{l}\text { Diabetes } \\
\text { Yes } \\
\text { No }\end{array}$ & $\begin{array}{l}35.6 \%(16) \\
64.4 \%(29)\end{array}$ & $\begin{array}{c}27.3 \%(24) \\
72.7 \%(9)\end{array}$ & 0.44 \\
\hline \multicolumn{4}{|l|}{ Lab values } \\
\hline Total testosterone & $526.04 \pm 128.49$ & $481.97 \pm 139.11$ & 0.15 \\
\hline$\overline{\mathrm{FSH}}$ & $6.48 \pm 1.96$ & $6.16 \pm 2.09$ & 0.49 \\
\hline$\overline{\mathrm{LH}}$ & $6.13 \pm 2.72$ & $4.93 \pm 2.72$ & 0.06 \\
\hline$\overline{\mathrm{PRL}}$ & $11.43 \pm 8.32$ & $12.11 \pm 4.90$ & 0.67 \\
\hline Fasting blood glucose & $106.80 \pm 29.60$ & $104.72 \pm 27.00$ & 0.75 \\
\hline Total cholesterol & $186.26 \pm 28.61$ & $201.60 \pm 30.67$ & 0.02 \\
\hline$\overline{\mathrm{LDL}}$ & $142.68 \pm 29.69$ & $140.51 \pm 25.76$ & 0.17 \\
\hline$\overline{\text { AST }}$ & $33.35 \pm 8.28$ & $27.06 \pm 8.78$ & 0.002 \\
\hline$\overline{\text { ALT }}$ & $36.82 \pm 9.17$ & $30.81 \pm 9.22$ & 0.006 \\
\hline$\overline{\text { GGT }}$ & $47.31 \pm 7.55$ & $38.03 \pm 10.94$ & 0.0001 \\
\hline
\end{tabular}

Figure 2.

SEP-2 and SEP-3 answers before and after three months of treatment with Tadalafil (5 mg/daily) plus nutritional supplement (1 cpr/daily), Group A, or Tadalafil (5 mg/daily) plus placebo, Group B. Both treatments significantly increased SEP-2 and SEP-3 in each group compared to baseline.

Data were analyzed by one-way ANOVA followed by Bonferroni post-test. There was no significant difference between group $A$ and group $B$.

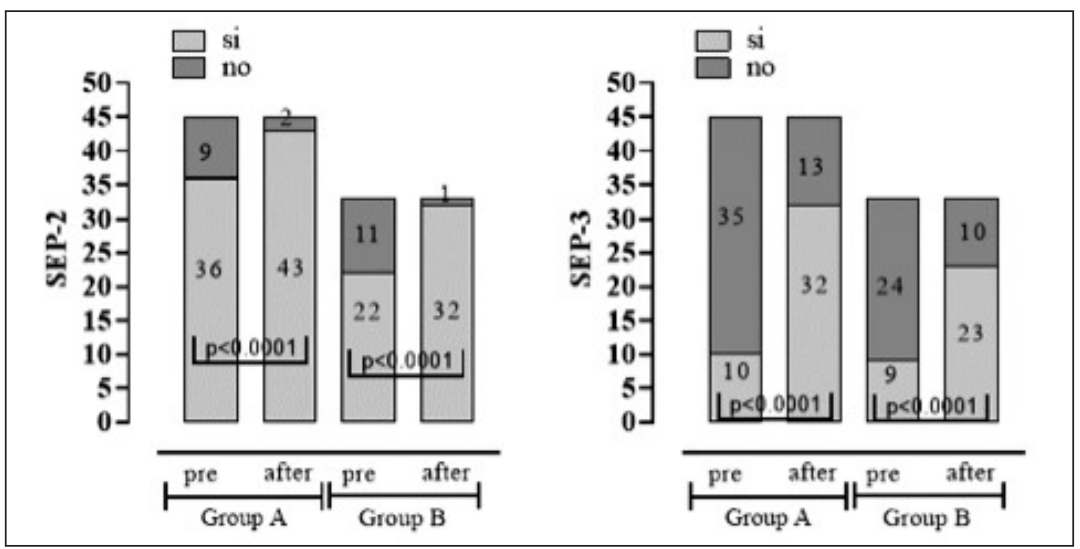

Table 2.

Hormonal levels before and after treatment.

\begin{tabular}{|l|ccc|ccc|}
\hline & Group A (before) & Group A (after) & P value & Group B (before) & Group B (after) & P value \\
\hline Total testosterone & $526.04 \pm 128.49$ & $532.34 \pm 115.73$ & 0.82 & $481.97 \pm 139.11$ & $485.57 \pm 122.48$ & 0.89 \\
\hline FSH & $6.48 \pm 1.96$ & $6.50 \pm 1.77$ & 0.96 & $6.16 \pm 2.09$ & $6.19 \pm 2.15$ & 0.95 \\
\hline LH & $6.13 \pm 2.72$ & $6.38 \pm 2.62$ & 0.68 & $4.93 \pm 2.72$ & $5.01 \pm 2.62$ & 0.89 \\
\hline PRL & $11.43 \pm 8.32$ & $12.61 \pm 4.71$ & 0.46 & $12.11 \pm 4.90$ & $12.39 \pm 4.71$ & 0.80 \\
\hline
\end{tabular}

Figure 1.

IIEF-5 score before and after three months of treatment with Tadalafil ( $5 \mathrm{mg} /$ daily) plus nutritional supplement (1 cpr/daily), Group A, or Tadalafil (5 mg/daily) plus placebo, Group B.

Both treatments significantly increased IIEF-5 score in each group compared to baseline

(Group A: $13.18 \pm 3.75$ vs $20.48 \pm 2.24, p<0.0001$;

Group B: $14.15 \pm 4.09$ vs $19.06 \pm 4.36, p<0.0001$ ).

The treatment with Tadalafil plus nutritional supplement (Group A) significantly increased IEEF-5 score compared to Tadalafil plus placebo (Group B) $(p<0.0001 ;+7.27 \pm 2.2$ and $4.9 \pm 2.79$, respectively).

Data expressed as mean \pm s.d. were analyzed by one-way ANOVA followed by Bonferroni post-test.

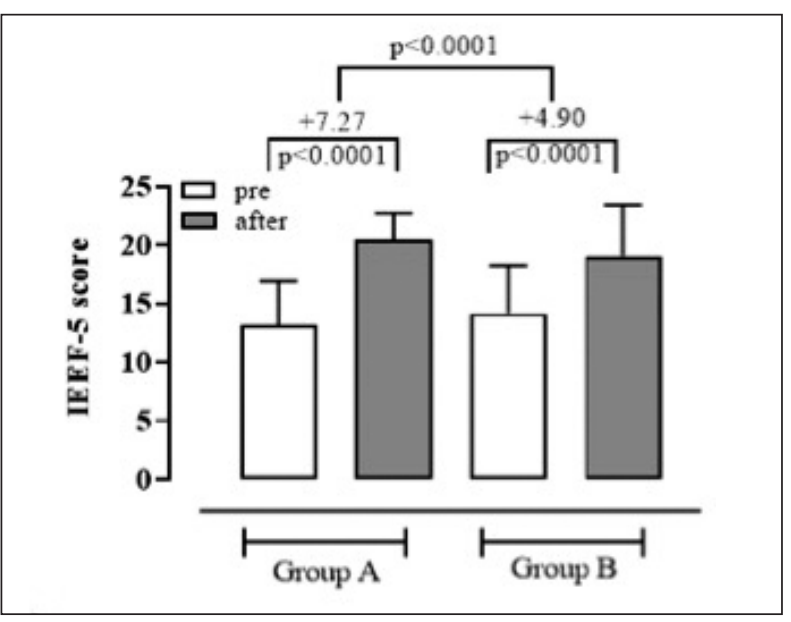


Table 3.

Adverse events.

\begin{tabular}{|l|c|c|c|}
\hline Adverse event & Group A & Group B & P value \\
\hline Headache & 6 & 4 & \\
\hline Nasal congestion & 1 & 3 & \\
\hline Back pain & 3 & 1 & \\
\hline Dyspepsia & 0 & 1 & \\
\hline Myalgia & 1 & 1 & \\
\hline Cough & 0 & 1 & \\
\hline Insomnia & 1 & 0 & \\
\hline Flushing & 6 & 5 & \\
\hline Dizziness & 2 & 2 & \\
\hline Total of adverse events reported & 20 & 18 & 0.38 \\
\hline
\end{tabular}

Figure 3.

Effect of Tadalafil (5 mg/daily) plus nutritional supplement (1 cpr/daily) on platelet cGMP of ED patients.

The levels of cGMP, expressed as pmoles $/ \mathrm{ml}$, were measured in $5 \times 105 / \mu \mathrm{l}$ platelet following stimulation with vehicle $(V)$ or diethylamine (DEA)-NONOate $(10$, and $100 \mu \mathrm{M})$. The cGMP content was evaluated before $(\cdot)$ or after one month of treatment (o). cGMP accumulation after treatment was significantly higher in platelets stimulated with DEANONOate $100 \mu \mathrm{M}$ compared to the same concentration of DEA-NONOate before the treatment $\left({ }^{*} p<0.05\right)$.

Data expressed as mean \pm s.e.m. were analyzed by one-way ANOVA followed by Bonferroni post-test.

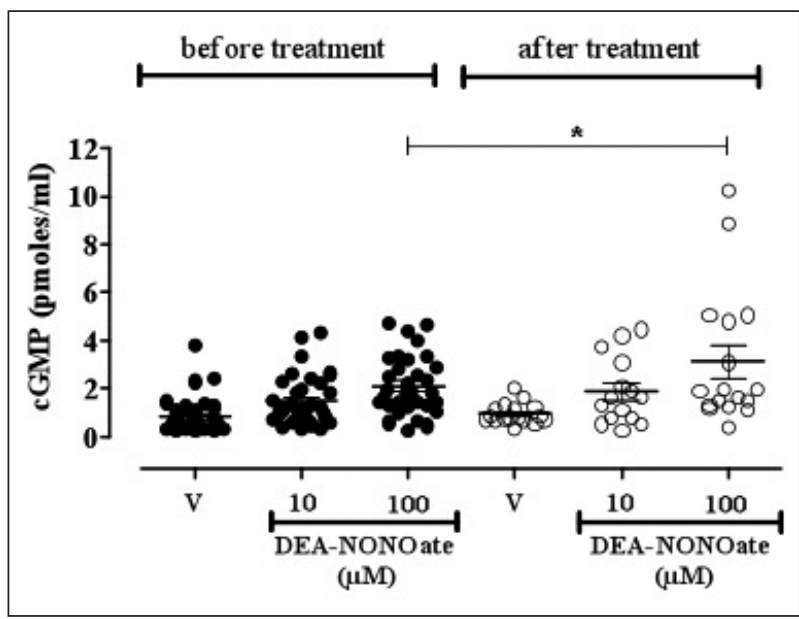

oleifera and rutin) $(\mathrm{p}<0.05)$ increased the cGMP content in platelets stimulated with DEA-NONOate $100 \mu \mathrm{M}$ compared with the same concentration of DEANONOate before the treatment i.e. at baseline (Figure 3).

\section{Discussion}

A variety of natural products, including isolated compounds from plants, have been tested for treatment of male sexual dysfunction (21). Although guidelines do not give any specific recommendation for their use, natural extracts are potentially useful in the management and treatment of male sexual dysfunction (16). Ginseng has been tested for its therapeutic properties, which include improving sexual function (22), physical performance (23), treating cancer (24), diabetes (25) and hypertension (26). Data available suggest that ginseng has some testosterone-like effects and it could contribute to smooth muscle relaxation of the corpus cavernosum via $\mathrm{NO}$ pathway (27).

Moringa oleifera has been long used in traditional medicine. Many studies have reported its antioxidant, hypoglycaemic, anti-dyslipidaemia activities, tissue-protective (liver, kidneys, heart, testes, and lungs), analgesic, antihypertensive and immunomodulatory actions (28-30). Rutin is a flavonoid glycoside characterized by antioxidant, antidiabetic, anti-lipid peroxidation actions. In particular, data suggest that rutin has antioxidant activity and increases testosterone levels in diabetic condition in preclinical studies. Furthermore, it has been shown that in vitro rutin can inhibit PDE5 and arginase increasing the availability of NO and cGMP (31-33). This nutritional supplement formulation, containing a balanced content of Moringa oleifera, rutin and ginseng, has designed to act as an endothelial protector to be used as an adjuvant in the treatment of ED. The efficacy of the nutritional supplement has been tested in a clinical study by performing a combination therapy with a low dose of chronic Tadalafil regimen that has shown to improve the erectile function in vasculogenic patients. There were no significant differences between the groups in terms of baseline erectile function and presence of comorbidities.

The addition of a daily capsule of the nutritional supplement to Tadalafil $5 \mathrm{mg}$ daily did not affect total testosterone, FSH, LH and PRL values. Although several animal and human studies suggested metabolic effects of Moringa oleifera and rutin, in our study,we did not found anti-dyslipidemic and hypoglycemic activity, probably because this was a chronic effects and 3 months could be a limited time to observe the effect. In addition, human studies showed that Moringa oleifera mainly determines a reduced post-prandial blood glucose levels and a long term reduction of $\mathrm{HbAlC}$, rather than fasting blood glucose and we did not assess these parameters as it was not the purpose of our study (34). The clinic al evaluation indicates a statistically significant effect on sexual function. The IIEF-5 score increased in group A of 7.27 points vs 4.90 in group B with a significant difference of 2.37 points that represents a $20 \%$ increase over the placebo treatment. There was no significant difference in SEP2 and SEP3 values between Tadalafil plus nutritional supplement and Tadalafil plus placebo. Thus, the treatment with the formulation (Panax ginseng, Moringa oleifera and rutin) improves the IEEF-5 score. The second part of the study was performed to validate the data obtained by using the questionnaires. Indeed, as in this case, questionnaires are strongly biased by the placebo treatment. We have previously shown that platelet cGMP represents a suitable and objective biomarker of PDE5-inhibitors efficacy in ED clinical studies.

This evidence relies on the fact that i) PDE5-inhibitors act by enhancing the NO/cGMP signaling ii) PDE5 is present in human platelets $(35,36)$ iii) treatment with PDE5inhibitors increases platelet cGMP levels $(18,37)$. In particular, we have demonstrated that, following chronic treatment with vardenafil $5 \mathrm{mg}$ /daily of ED patients, the platelet cGMP levels were significantly increased and well correlated (significantly) with the VSS-Rigiscan measure- 
ment (18). Thus, the measurement of platelet cGMP from blood samples of patients represents an unbiased marker of activity. The analysis of platelets harvested from patients treated with the nutritional supplement plus Tadalafil showed a significant increase in the cGMP levels when stimulated with DEA-NONOate $100 \mu \mathrm{M}$. This result suggests that the treatment with the nutritional supplement ameliorates and extends the activity of the chronic treatment with Tadalafil maintaining a significant more elevated levels of inhibition of PDE5. In this context, it is important to stress that there are several clinical pieces of evidence that PDE5-inhibitors effect can go beyond their half-life $(18,38)$. Indeed, clinical data reported that men still have facilitated erections when the levels of PDE5-inhibitors are below of the therapeutic plasmatic concentration (39-41).

\section{Conclusions}

In conclusion, the significant increase of the IIEF-5 can be ascribed to the nutritional supplement properties. Indeed, beyond the well-known antioxidant effects, Moringa oleifera, ginseng and rutin, it has been reported that can enhance the endothelial NO and cGMP production (41-43).

\section{References}

1. Shamloul R, Ghanem H. Erectile dysfunction. Lancet. 2013; 381;153-165.

2. Gareri P, Castagna A, Francomano D, et al. Erectile dysfunction in the elderly: an old widespread issue with novel treatment perspectives. Int J Endocrinol. 2014; 2014:878670.

3. Yafi FA, Jenkins L, Albersen M, et al. Erectile dysfunction. Nat Rev Dis Primers. 2016; 2:16003.

4. Baumann F, Hehli D, Makaloski V, et al. Erectile dysfunctionoverview from a cardiovascular perspective. Vasa. 2017; 10:1-7.

5. Eisenberg ML, Meldrum D. Effects of age on fertility and sexual function. Fertil Steril. 2017; 107:301-4.

6. Nguyen HMT, Gabrielson AT, Hellstrom WJG. Erectile dysfunction in young men-a review of the prevalence and risk factors. Sex Med Rev. 2017; 5:508-20.

7. Hsu B, Hirani V, Naganathan $V$, et al. Sexual function and mortality in older men: the concord health and ageing in men project. J Gerontol A Biol Sci Med Sci. 2017; 72:520-7.

8. Dean RC, Lue TF. Physiology of penile erection and pathophysiology of erectile dysfunction. Urol Clin North Am. 2005; 32:379-95.

9. Andersson KE. Mechanisms of penile erection and basis for pharmacological treatment of erectile dysfunction. Pharmacol Rev. 2011; 63:811-59.

10. Kass DA, Takimoto E, Nagayama T, Champion HC. Phosphodiesterase regulation of nitric oxide signaling. Cardiovasc Res. 2007; 75:303-14

11. Allen MS, Walter EE. Erectile dysfunction: an umbrella review of meta-analyses of risk-factors, treatment, and prevalence outcomes. J Sex Med. 2019; 16:531-541.

12. Karatza AA, Bush A, Magee AG. Safety and efficacy of sildenafil therapy in children with pulmonary hypertension. Int J Cardiol. 2005; 100:267-273.
13. Lewis RJ, Johnson RD, Blank CL. Quantitative determination of sildenafil (Viagra) and its metabolite (UK-103,320) in fluid and tissue specimens obtained from six aviation fatalities. J Anal Toxicol. 2006; 30:14-20.

14. Palmieri A, Arcaniolo D, Palumbo F, et al. Low intensity shockwave therapy in combination with phosphodiesterase-5 inhibitors is an effective and safe treatment option in patients with vasculogenic ED who are PDE5i non-responders: a multicenter single-arm clinical trial; SIA-Low intensity shock wave for Erectile Dysfunction (LED) Study Group. Int J Impot Res. 2020 Jul 18 doi: 10.1038/s41443-0200332-7.

15. Corona G, Rastrelli G, Burri A, et al. First-generation phosphodiesterase type 5 inhibitors dropout: a comprehensive review and meta-analysis. Andrology. 2016; 4:1002-1009.

16. Srivatsav A, Balasubramanian A, Pathak UI, et al. Efficacy and safety of common ingredients in aphrodisiacs used for erectile dysfunction: a review. Sex Med Rev. 2020; 8:431-442.

17. Rosen RC, Riley A, Wagner G, et al. The International Index of Erectile Function (IIEF): a multidimensional scale for assessment of erectile dysfunction. Urology. 1997; 49:822-30.

18. Mirone V, d'Emmanuele di Villa Bianca R, Mitidieri E, et al. Platelet cyclic guanosine monophosphate as a biomarker of phosphodiesterase type 5 inhibitor efficacy in the treatment of erectile dysfunction: a randomized placebo-controlled study. Eur Urol. 2009; 56:1067-73

19. d'Emmanuele di Villa Bianca R, Mitidieri E, Mirone V, et al. An ex vivo standardized assay to measure human platelet cGMP. J Pharmacol Toxicol Methods. 2011; 64:164-7.

20. Keefer LK, Nims RW, Davies KM, Wink DA. NONOates" (1-substituted diazen-1-ium-1, 2-diolates) as nitric oxide donors: convenient nitric oxide dosage forms. Methods Enzymol. 1996; 268:281-93.

21. Malviya N, Malviya S, Jain S, Vyas S. A review of the potential of medicinal plants in the management and treatment of male sexual dysfunction. Andrologia. 2016; 48:880-93.

22. Jang DJ, Lee MS, Shin BC, et al. Red ginseng for treating erectile dysfunction: a systematic review. Br J Clin Pharmacol. 2008; $66: 444-450$

23. Kulaputana O, Thanakomsirichot S, Anomasiri W. Ginseng supplementation does not change lactate threshold and physical performances in physically active Thai men. J Med Assoc Thai. 2007; 90:1172-1179.

24. Helms S. Cancer prevention and therapeutics: Panax ginseng. Altern Med Rev. 2004; 9:259-274.

25. Kim S, Shin BC, Lee MS, et al. Red ginseng for type 2 diabetes mellitus: a systematic review of randomized controlled trials. Chin J Integr Med. 2011; 17:937-944.

26. Rhee MY, Kim YS, Bae JH, et al. Effect of Korean red ginseng on arterial stiffness in subjects with hypertension. J Altern Complement Med. 2011; 17:45-49.

27. de Andrade E, de Mesquita AA, Claro Jde A, et al. Study of the efficacy of Korean Red Ginseng in the treatment of erectile dysfunction. Asian J Androl. 2007; 9:241-4.

28. Atawodi SE, Atawodi JC, Idakwo GA, et al. Evaluation of the polyphenol content and antioxidant properties of methanol extracts of the leaves, stem, and root barks of Moringa oleifera Lam. J Med Food. 2010; 13:710-716.

29. Jaiswal D, Kumar RP, Kumar A, et al. Effect of Moringa oleifera Lam leaves aqueous extract therapy on hyperglycemic rats. J Ethnopharmacol. 2009; 123:392-396. 
30. Jung IL. Soluble extract of Moringa oleifera leaves with a new anticancer activity. PLOSOne 2014;9:e95492

31. Al-Roujeaie AS, Abuohashish HM, Ahmed MM, Alkhamees OA. Effect of rutin on diabetic-induced erectile dysfunction: Possible involvement of testicular biomarkers in male rats. Andrologia. 2017; 49

32. Oboh $G$, Adebayo AA, Ademosun AO, Boligon AA. In vitro inhibition of phosphodiesterase -5 and arginase activities from rat penile tissue by two Nigerian herbs (Hunteria umbellata and Anogeissus leiocarpus). J Basic Clin Physiol Pharmacol. 2017; 28:393-401.

33. Zhang Y, Huang C, Liu S, et al. Effects of quercetin on intracavernous pressure and expression of nitrogen synthase isoforms in arterial erectile dysfunction rat model. Int J Clin Exp Med. 2015; 8:7599-605.

34. Nova E, Redondo-Useros N, Martínez-García RM, et al. Potential of Moringa oleifera to improve glucose control for the prevention of diabetes and related metabolic alterations: a systematic review of animal and human studies. Nutrients. 2020; 12:2050.

35. Beavo JA. Cyclic nucleotide phosphodiesterases: functional implications of multiple isoforms. Physiol Rev. 1995; 75:725-48.

36. Wallis RM, Corbin JD, Francis SH, Ellis P. Tissue distribution of phosphodiesterase families and the effects of sildenafil on tissue cyclic nucleotides, platelet function, and the contractile responses of trabeculae carneae and aortic rings in vitro. Am J Cardiol. 1999; 83:3C-12C.
37. Dunkern TR, Hatzelmann A. The effect of sildenafil on human platelet secretory function is controlled by a complex interplay between phosphodiesterases 2, 3 and 5. Cell Signal. 2005; 17:331-9.

38. Francis SH, Morris GZ, Corbin JD. Molecular mechanisms that could contribute to prolonged effectiveness of PDE5 inhibitors to improve erectile function. Int J Impot Res. 2008; 20:333-42.

39. Moncada I, Jara J, Subirà D, et al. Efficacy of sildenafil citrate at 12 hours after dosing: re-exploring the therapeutic window. Eur Urol. 2004; 46:357-61.

40. Young JM, Feldman RA, Auerbach SM, et al. Tadalafil improved erectile function at twenty-four and thirty-six hours after dosing in men with erectile dysfunction: US trial. J Androl. 2005; 26:310-8.

41. Shabsigh R, Seftel AD, Rosen RC, et al. Review of time of onset and duration of clinical efficacy of phosphodiesterase type 5 inhibitors in treatment of erectile dysfunction. Urology. 2006; 68:689-96.

42. Direk Aekthammarat, Panot Tangsucharit, Patchareewan Pannangpetch, Thanaporn Sriwantana, Nathawut Sibmooh. Moringa oleifera leaf extract enhances endothelial nitric oxide production leading to relaxation of resistance artery and lowering of arterial blood pressure. Biomed Pharmacother. 2020; 130:110605.

43. Nandave M, Ojha SK, Joshi S, et al. Moringa oleifera leaf extract prevents isoproterenol-induced myocardial damage in rats: Evidence for an antioxidant, antiperoxidative, and cardioprotective intervention. Journal of Medicinal Food. 2009; 12:47-55.

\section{Correspondence}

Vincenzo Mirone, MD

Luigi Napolitano, MD (Corresponding Author)

nluigi89@libero.it

Roberto La Rocca, MD

robertolarocca87@gmail.com

Giuseppe Celentano, MD

Department of Neurosciences, Sciences of Reproduction, and Odontostomatology, University of Naples Federico II

Via Sergio Pansini 5, 80131 Naples (Italy)

Roberta D'Emmanuele di Villa Bianca, MD

Emma Mitidieri, MD

Raffaella Sorrentino, $M D$

Domenico Vanacore, MD

Carlotta Turnaturi, MD

Giuseppe Cirino, MD

Department of Pharmacy, School of Medicine and Surgery, University of Naples, Federico II, Via D. Montesano, 49, 80131 Naples (Italy)

Arianna Vanelli, MD

Responsabile Ricerca e Sviluppo Nutrilinea SRL, Varese (Italy)

Davide Arcaniolo, MD

Urology Unit, Department of Woman Child and of General and Specialist

Surgery, University of Campania "Luigi Vanvitelli", 80131 Naples (Italy) 